\title{
The Topological Indices of the Non-commuting Graph for Symmetric Groups
}

\author{
Nur Idayu Alimon ${ }^{1^{*}}$, Nor Haniza Sarmin ${ }^{1}$ and Ahmad Erfanian ${ }^{2}$ \\ ${ }^{1}$ Department of Mathematical Sciences, Faculty of Science, Universiti Teknologi Malaysia, Johor \\ Baru \\ ${ }^{2}$ Department of Pure Mathematics, Faculty of Mathematical Sciences, Ferdowsi University of \\ Mashhad, Mashhad, Iran
}

\begin{abstract}
Topological indices are the numerical values that can be calculated from a graph and it is calculated based on the molecular graph of a chemical compound. It is often used in chemistry to analyse the physical properties of the molecule which can be represented as a graph with a set of vertices and edges. Meanwhile, the non-commuting graph is the graph of vertex set whose vertices are non-central elements and two distinct vertices are joined by an edge if they do not commute. The symmetric group, denoted as $S_{n}$, is a set of all permutation under composition. In this paper, two of the topological indices, namely the Wiener index and the Zagreb index of the non-commuting graph for symmetric groups of order 6 and 24 are determined.
\end{abstract}

Keywords: Wiener index; Zagreb index; non-commuting graph; symmetric groups

\section{INTRODUCTION}

A topological index is a single number that can be used to characterize the graph of a molecule (Hosoya, 1971). It appears to be a convenient device for converting chemical constitution into a number and this number must have the same value regardless of ways in which the corresponding graph is drawn or labelled (Trinajstic̀, 1983).

Most of the proposed topological indices involve either the distance between two vertices or the connectivity which means the degree of the vertex. In mathematical chemistry, the molecular graph which consists of the atoms and bonds can be represented as the graph in graph theory where the atoms represent the set of vertices and bonds represent the set of edges of a graph. The topological indices of the molecular structure have been determined by many researchers in order to apply in some models for solving real world problem.

In mathematical approach, various topological indices of a simple graph have been studied but this paper extend it to a specific group which is symmetric group. This approach has many advantages which include the simplicity and rapidity of the calculation to find the physicochemical properties of the molecular structure. This paper focuses on the Wiener index, the first Zagreb index and the second Zagreb index of the noncommuting graph of the symmetric groups and it consists of three sections. The first section is the introduction, followed by the preliminaries where some basic concepts and definitions on group theory and graph theory are stated. In the last section, our main results are presented.

\section{PRELIMINARIES}

In this section, some basic concepts and definitions in group theory, graph theory and topological indices are included. This research focuses on symmetric group, $S_{n}$ which is the group of permutations on a set with $n$ elements. Some definitions in group theory and graph theory that are used in this research are listed in the following.

Definition 1 (Raza \& Faizi, 2013) Non-commuting Graph Let $G$ be a finite group. The non-commuting graph of $G$,

*Corresponding author's e-mail: nuridayualimon@yahoo.com 
denoted by $\Gamma_{G}$, is the graph of vertex set $G-Z(G)$ and two distinct vertices $x$ and $y$ are joined by an edge whenever $x y \neq$ $y x$.

Based on the graph, the connectivity of the graph which is the degree of the vertex and the distance between two vertices can be determined. The degree of the vertex is defined as the number of edges that are connected to the vertex, while the distance between two vertices is the shortest path from one vertex to the other.

The Wiener index was first introduced by Harold Wiener in 1947 and used it to determine the physical properties of alkanes, specifically in determining the boiling point of alkanes (Wiener, 1947) while Gutman and Trinajstic introduced the Zagreb index in 1972 where it is divided into the first Zagreb index and the second Zagreb index which known as the approximate formula for the total $\pi$-energy of a graph (Gutman \& Trinajstic̀, 1972).

In 2012, Mizargar and Ashrafi have generalized some indices for a non-commuting graph specifically for dihedral groups and semi-dihedral groups (Mizargar \& Ashrafi, 2012) while Vijayabarathi and Anjaneyulu have extended the research on the applications of the Wiener index in analysing the Wiener index of the structure of organic molecules (Vijayabarathi \& Anjaneyulu, 2013).

In the following, the definitions of three types of topological indices of a graph for a finite group are presented, starting with the Wiener index.

Definition 2 (Wiener, 1947) The Wiener Index

Let $v_{i}$ and $v_{j}$ be two distinct vertices where $i \neq j$ and $\Gamma$ be the connected graph with $m$ vertices. The Wiener index of the graph is defined as half of the sum of the distances between every pair of vertices of $\Gamma$, written as:

$$
W(\Gamma)=\frac{1}{2} \sum_{i=1}^{m} \sum_{j=1}^{m} d\left(v_{i}, v_{j}\right),
$$

where $d\left(v_{i}, v_{j}\right)$ is the distance between vertex $i$ and vertex $j$.

Next, following are the definition of the first Zagreb index and the second Zagreb index.

Definition 3 (Gutman \& Trinajstic̀, 1972) The First Zagreb Index
Let $\Gamma$ be a connected graph. Then, the first Zagreb index is the sum of squares of the degrees of the vertices of, written as:

$$
M_{1}(\Gamma)=\sum_{v \in V(\Gamma)} \operatorname{deg}(v)^{2},
$$

where $\operatorname{deg}(v)$ is the number of edges connected to vertex $v$.

Definition 4 (Gutman \& Trinajstic̀, 1972) The Second Zagreb Index

Let $\Gamma$ be a connected graph. Then, the second Zagreb index is the sum of the product of the degrees of pair of adjacent vertices of $\Gamma$, written as:

$$
M_{2}(\Gamma)=\sum_{u, v \in E(\Gamma)} \operatorname{deg}(u) \operatorname{deg}(v),
$$

where $u, v$ are the vertices on the edge connected them.

\section{RESULTS AND DISCUSSIONS}

In this section, the Wiener index, the first Zagreb index and the second Zagreb index of the non-commuting graph for symmetric groups of order six and 24 are determined.

\section{Proposition 5}

Let $\Gamma$ be the non-commuting graph and $S_{3}$ is the symmetric group of order 6 . Then, the non-commuting graph of the symmetric group of order 6 is found in the following.

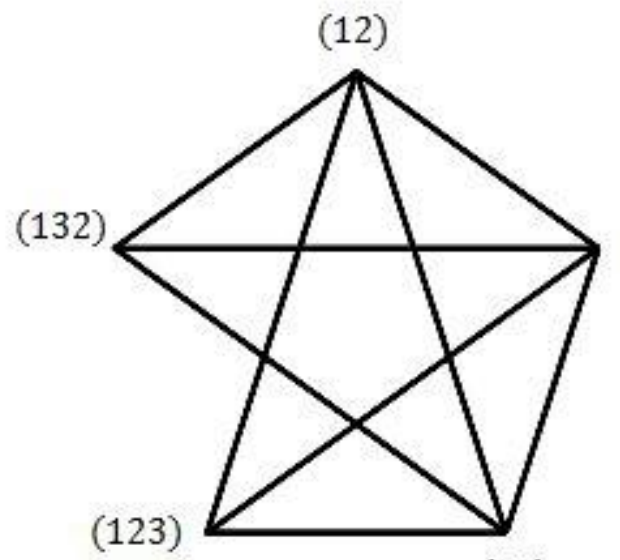

Figure 1. The non-commuting graph of $S_{3}$

\section{Proof}

The symmetric group of order six, $S_{3}$ has six elements which consists only one element in the center, namely (1). By 
Definition 1, the set of vertices of the non-commuting graph is the set of non-central elements where two vertices are connected if they do not commute. Hence, the noncommuting graph of $S_{3}$ consists of five vertices and nine edges as depicted in Figure 1.

\section{Proposition 6}

Let $\Gamma$ be the non-commuting graph and $S_{4}$ is the symmetric group of order 24. Then, the non-commuting graph of the symmetric group of order 24 is guven in Figure 2.

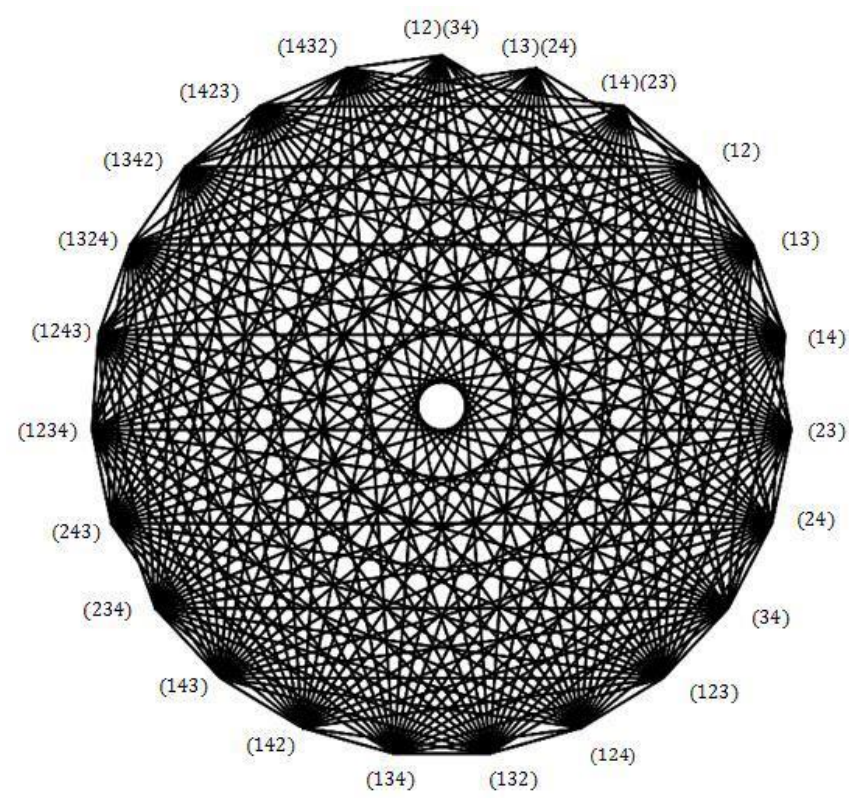

Figure 2. The non-commuting graph of $S_{4}$

\section{Proof}

The symmetric group of order 24, $S_{4}$ has 24 elements which consists only one element in the center, namely (1). By Definition 1, the non-commuting graph of $S_{4}$ consists of 23 vertices and 228 as shown in Figure 2.

\section{A. The Topological Indices of the Non-commuting

$$
\text { Graph of } S_{3}
$$

In this subsection, the topological indices of the noncommuting graph for symmetric group of order six are computed. The following propositions give the Wiener index, the first Zagreb index and the second Zagreb index of the noncommuting graph of $S_{3}$.

\section{Proposition 7}

Let $G$ be the symmetric group of order six, $S_{3}$ and $\Gamma_{G}$ is the non-commuting graph of $G$. Then, the Wiener index of the non-commuting graph of , :

$$
W\left(\Gamma_{G}\right)=11 .
$$

\section{Proof}

Since any two elements in $S_{3}$ do not commute except for (123) and (132), there are only two vertices that will have the distance two, then the Wiener index is found as in the following:

$$
\begin{aligned}
W\left(\Gamma_{G}\right)= & \frac{1}{2} \sum_{i=1}^{5} \sum_{j=1}^{5} d(i, j) \\
= & \frac{1}{2} \sum_{i=1}^{5}[d(i, 1)+d(i, 2)+\cdots+d(i, 5)] \\
= & \frac{1}{2}[(d(1,1)+d(1,2)+\cdots d(1,5))+\cdots+ \\
& (d(5,1)+d(5,2)+\cdots+(d(5,5))] \\
= & \frac{1}{2}[(0+1+1+1+1)+\cdots+ \\
& (1+1+1+2+0)] \\
= & 11,
\end{aligned}
$$

where $i, j$ are the vertices of $\Gamma_{G}$.

\section{Proposition 8}

Let $G$ be the symmetric group of order six, $S_{3}$ and $\Gamma_{G}$ is the non-commuting graph of $G$. Then, the first Zagreb index of the non-commuting graph of $G$ :

$$
M_{1}\left(\Gamma_{G}\right)=66 .
$$

Proof. Since any two elements in $S_{3}$ do not commute except for (123) and (132), there are only two vertices that will have degree 3 and the other vertices have degree 4 , then the first Zagreb index is in the following:

$$
\begin{aligned}
M_{1}\left(\Gamma_{G}\right) & =\sum_{v \in V(\Gamma)} \operatorname{deg}(v)^{2} \\
& =\sum_{i=1}^{5} \operatorname{deg}(i)^{2} \\
& =\operatorname{deg}(1)^{2}+\operatorname{deg}(2)^{2}+\cdots+\operatorname{deg}(5)^{2} \\
& =4^{2}+4^{2}+4^{2}+3^{2}+3^{2} \\
& =66,
\end{aligned}
$$

where $i$ denotes a vertex of $\Gamma_{G}$.

\section{Proposition 9}

Let $G$ be the symmetric group of order six, $S_{3}$ and $\Gamma_{G}$ is the non-commuting graph of $G$. Then, the second Zagreb index of the non-commuting graph of $G$,

$$
M_{2}\left(\Gamma_{G}\right)=120 .
$$




\section{Proof}

Since the non-commuting graph of $S_{3}$ is not a complete graph, then the degree of each vertex of the graph is not the same. Hence, there are three edges that have both vertex of degree 4 and six edges that have vertex 4 and 3. The second Zagreb index is in the following:

$$
\begin{aligned}
M_{2}\left(\Gamma_{G}\right)= & \sum_{e \in E(\Gamma)} \operatorname{deg}(u)^{2} \operatorname{deg}(v)^{2} \\
= & \sum_{i=1}^{9} e_{i} \\
= & e_{1}+e_{2}+\cdots+e_{9} \\
= & \operatorname{deg}(1) \operatorname{deg}(2)+\operatorname{deg}(2) \operatorname{deg}(3)+\cdots+ \\
& \operatorname{deg}(3) \operatorname{deg}(5) \\
= & 120,
\end{aligned}
$$

where $i$ denotes the edge of $\Gamma_{G}$.

\section{B. The Topological Indices of the Non-commuting}

$$
\text { Graph of } S_{4}
$$

In this subsection, the topological indices of the noncommuting graph of symmetric group of order 24 are computed. The following lemmas are the Wiener index, the first Zagreb index and the second Zagreb index of the noncommuting graph of $S_{4}$.

\section{Proposition 10}

Let $G$ be the symmetric group of order $24, S_{4}$ and $\Gamma_{G}$ is the non-commuting graph of $G$. Then, the Wiener index of the non-commuting graph of , :

$$
W\left(\Gamma_{G}\right)=278 .
$$

\section{Proof}

In $S_{4}$, the elements with length two and length four do not commute with only two other elements, the elements with length three do not commute with only the other one element which is its inverse, and the element with disjoint cycle with length two each do not commute with the other six elements. Hence, the Wiener index of the non-commuting graph of $S_{4}$ is in the following:

$$
\begin{aligned}
W\left(\Gamma_{G}\right)= & \frac{1}{2} \sum_{i=1}^{23} \sum_{j=1}^{23} d(i, j) \\
& =\frac{1}{2} \sum_{i=1}^{23}[d(i, 1)+d(i, 2)+\cdots+d(i, 23)] \\
& =\frac{1}{2}[(d(1,1)+d(1,2)+\cdots d(1,23))+\cdots+
\end{aligned}
$$

$$
\begin{aligned}
& (d(23,1)+d(23,2)+\cdots+(d(23,23))] \\
= & \frac{1}{2}[(0+2+2+2+1+1+\cdots+2)+\cdots \\
& +(1+2+1+1+\cdots+0)] \\
= & 278,
\end{aligned}
$$

where $i, j$ are the vertices of $\Gamma_{G}$.

\section{Proposition 11}

Let $G$ be the symmetric group of order $24, S_{4}$ and $\Gamma_{G}$ is the non-commuting graph of $G$. Then, the first Zagreb index of the non-commuting graph of $G$,

$$
M_{1}\left(\Gamma_{G}\right)=9096 \text {. }
$$

\section{Proof}

Since all the elements of length two and length four have degree 20, the elements of length three have degree 21, and the elements of disjoint cycle with two elements length two have degree 16, the first Zagreb index is in the following:

$$
\begin{aligned}
M_{1}\left(\Gamma_{G}\right) & =\sum_{v \in V(\Gamma)} \operatorname{deg}(v)^{2} \\
& =\sum_{i=1}^{23} \operatorname{deg}(i)^{2} \\
& =\operatorname{deg}(1)^{2}+\operatorname{deg}(2)^{2}+\cdots+\operatorname{deg}(23)^{2} \\
& =16^{2}+16^{2}+16^{2}+20^{2}+\cdots+20^{2} \\
& =9096,
\end{aligned}
$$

where $i$ denotes a vertex of $\Gamma_{G}$.

\section{Proposition 12}

Let $G$ be the symmetric group of order $24, S_{4}$ and $\Gamma_{G}$ is the non-commuting graph of $G$. Then, the second Zagreb index of the non-commuting graph of $G$,

$$
M_{2}\left(\Gamma_{G}\right)=75,233 .
$$

\section{Proof}

Since the non-commuting graph of $S_{4}$ is not a complete graph, then the degree of each vertex is not the same. Hence, there are 60 edges that have both vertex with degree 20, 96 edges that have vertex of degree 20 and 21, 24 edges that have vertex of degree 20 and 16, 24 edges that have both vertex of degree 21, and 24 edges that have vertex of degree 21 and 16. Hence, the second Zagreb index of the non-commuting graph of $S_{4}$ is in the following:

$$
\begin{aligned}
M_{2}\left(\Gamma_{G}\right) & =\sum_{e \in E(\Gamma)} \operatorname{deg}(u)^{2} \operatorname{deg}(v)^{2} \\
& =\sum_{i=1}^{228} e_{i}
\end{aligned}
$$




$$
\begin{aligned}
= & e_{1}+e_{2}+\cdots+e_{228} \\
= & \operatorname{deg}(1) \operatorname{deg}(5)+\operatorname{deg}(2) \operatorname{deg}(4)+\cdots+ \\
& \operatorname{deg}(23) \operatorname{deg}(15) \\
= & 16(20)+16(20)+\cdots+20(20) \\
= & 75,233,
\end{aligned}
$$

where $i$ denotes the edge of $\Gamma_{G}$.

\section{SUMMARY}

In this paper, the Wiener index, the first Zagreb index and the second Zagreb index of the non-commuting graph for symmetric groups of order six and 24 are computed. It is found that all of the topological indices are directly proportional to the order of the symmetric groups.

When the order of the symmetric groups is higher, the non-commuting graphs have more vertices which means the molecular structure is more complicated and has more branches. Hence, the structure is more compact which lead to the limited frequency of molecular collisions between atoms. So, it requires less heat to compensate the energy losses and boil at the lower temperature.

Therefore, in terms of boiling point of the structure, the higher the value of the Wiener index and Zagreb index, the lower the temperature of the structure.

\section{ACKNOWLEDGEMENTS}

This work financially supported by the Universiti Teknologi Malaysia (UTM) and the first author is indebted to UTM for her scholarship.

\section{REFERENCES}

Gutman, I. \& Trinajstic̀. N. 1972, Graph theory and molecular orbitals. Total П-electron energy of alternant hydrocarbon. Chemical Physics Letters, 17, 535-538.

Hosoya, H. 1971, Topological Index. A newly proposed quantity characterizing the topological nature of structural isomers of saturated hydro-carbons. Bulletin of Chemical Society of Japan, 44, 2332-2339.

Mizargar, M. \& Ashrafi, A.R. 2012, Some distance-based topological indices of a non-commuting graph. Hacettepe Journal of Mathematics and Statistics, 41, 515-526.

Raza, Z. \& Faizi, S. 2013, Non-commuting graph of finitely presented group. Scientific International (Lahore), 25, 883885.

Trinajstic̀. N. 1983, Chemical Graph Theory. CRC Press. p. 225.

Vijayabarathi, A. \& Anjaneyulu, G.S.G.N. 2013, Wiener index of a graph and chemical applications. International Journal of ChemTech Research, 5, 1847-1853.

Wiener, H. 1947, Structural determination of the paraffin boiling points. Journal of American Chemical Society, 69, 17-20. 